\title{
Startle decrement and secondary reinforcement stimulation'
}

HARVARD L. ARMUS AND DOHNA SHIADOWSKI-DOLIHSKY

THE UNIVERSITY OF TOLEDO
The following study was designed to test the hypothesis that the decrement in startle response to a percussive auditory stimulus, measured in the presence of an extraneous stimulus, is greater if the extraneous stimulus had been previously associated with receipt of food (i.e., is a secondary reinforcer). Two groups of food-deprived rats were given equal experience with a visual-auditory stimulus in a stabilimeter box, but for Group E, this stimulus was paired with food, while for Group $C$, it was not. All Ss received the startle stimulus, a loud "pop," for 8 trials a day over 3 test days, with the extraneous stimulus present on half the trials. Startle decrement in the presence of the extraneous stimulus was significantly greater for Group $E$ than for Group $C$ on Test Day $1(p<.02)$ and over Test Days $1-3(p<.025)$, confirming the hypothesis.

Armus et al (1964) presented evidence that the startle reaction to a percussive, auditory stimulus was reduced in the presence of an intermittent visualauditory secondary reinforcer. Previously Trapold (1962) failed to find this effect. However, Trapold employed startle stimulus adaptation trials, and also presented the startle stimulus during the secondary reinforcement training phase while in the Armus et al study, there was no opportunity for adaptation to the startle stimulus to occur before the extinction (test) sessions. (It should be noted that Trapold organized his study around the concept of incentive motivation, rather than secondary reinforcement.)

In 1963, Hoffman and Fleshler reported that intermittent white noise, per se, lowered the startle magnitude of rats to an auditory startle stimulus while steady white noise raised it. No differential effects were found for intermittent, steady, and absent light, as opposed to white noise. Although the startle stimulus, extraneous stimulus, and response measure were different from that used by Armus et al, it was decided to determine whether the reported startle suppression associated with the presence of the secondary reinforcer resulted from the secondary reinforcing qualities of the extraneous stimulus, or from its purely stimulus qualities.

\section{METHOD}

\section{Subjects and Apparatus}

Ss were 38 naive, male, albino rats, $102-111$ days old at the beginning of training. The apparatus, previously described (Armus et al, 1964), was a small plywood box fitted with a food cup and having a $6 \mathrm{w}$ clear lamp mounted on its transparentlid. The "firing" of a Super Nu-Matic paper punching pistol provided the startle stimulus, and $S$ 's startle response was transmitted to a recording pen by means of a thread and pulley system. The startle response was recorded in $\mathrm{mm}$. of pen deflection with no correction for arc distortion. The secondary reinforcement stimulus consisted of the repeated flashing of the aforementioned lamp and the associated clicking of a relay. The study was carried out in a sound shielded, dimly illuminated room of high accoustical reflectivity.

\section{Procedure}

Ss were housed in individual cages with water always available. Eleven to 12 days before the beginning of pre-training, Ss were put on a feeding schedule of $1 \mathrm{hr}$. access to dry Purina Laboratory Chow checkers every $24 \mathrm{hr}$. arranged so that Ss were approximately 20 to $23 \mathrm{hr}$. deprived when run. Ss received 2 days of pretraining, 16 days of training, and 3 days of testing.

Ss were divided into two groups, $E(N=20)$ and $\mathrm{C}(\mathrm{N}=18)$. For Group $\mathrm{E}$ on Pretraining Day 1 , the food cup in the stabilimeter box was baited with 15 45-mg. food pellets (P. J. Noyes Co.), and, when these had been consumed by the rat, 15 more were delivered manually, one at a time. Pretraining Day 2 was similar, except that the food cup was baited with only 1 pellet and a total of 49 pellets was delivered manually at $15-\mathrm{sec}$. intervals. On each of the 16 training days, each $S$ of Group $E$ received 20 food pellets in the stabilimeter box. The intertrial intervals (ITIs) were scheduled in 4 blocks of either $20,40,60$, or $80 \mathrm{sec}$. each, with 5 pellets being delivered in each ITI block. The order of ITI blocks was arranged in a Latin square over 4 training days, this Latin square being repeated 3 times over the 16 training days. At the beginning of each trial, the secondary reinforcement stimulus (flashing light and relay clicking) came on for 10 sec.; after 6 sec. of this stimulus, a food pellet was manually delivered to $S$. At the end of the 10-sec. period, the timing mechanism was automatically reset, and the next interval was begun. The procedure was identical for Group C, except that no food was delivered in the stabilimeter box, either during training or pre-training. Five min. before the daily feeding period, Ss of this group received, in their home cages, the same number of $45 \mathrm{mg}$. food pellets as $\mathrm{Ss}$ of Group $E$ had received in the stabilimeter box.

For Group E, testing differed from training in that a food pellet was no longer delivered on each trial, the startle stimulus (shot) being presented in its place. There were 3 test days with 8 test trials per day, 4 with the startle stimulus being presented at the usual time of food pellet delivery and the secondary 
Table 1. Mean Startle Deflection in $\mathrm{mm}$. for Experimental and Control Groups, Stimulus-Present and Stimulus-Absent Conditions

\begin{tabular}{|c|c|c|c|c|c|c|c|c|c|}
\hline \multirow[b]{2}{*}{ Group } & \multirow[b]{2}{*}{ Condition } & \multicolumn{2}{|c|}{ Day 1} & \multicolumn{2}{|c|}{ Day 2} & \multicolumn{2}{|l|}{ Test Doy } & \multicolumn{2}{|c|}{ Doys 1-3 } \\
\hline & & Mean Startie & Difference & Mean Startle & Difference & Mean Startle & Difference & Mean Startle & Difference \\
\hline$E:$ & $\begin{array}{l}\mathrm{Sr}^{r} \text { absent } \\
\mathrm{S}^{r} \text { present }\end{array}$ & $\begin{array}{l}34.11 \\
22.14\end{array}$ & 11.97 & $\begin{array}{l}31.69 \\
21.10\end{array}$ & 10.59 & $\begin{array}{l}26.93 \\
16.58\end{array}$ & 10.35 & $\begin{array}{l}30.99 \\
19.92\end{array}$ & 11.07 \\
\hline$C:$ & $\begin{array}{l}\text { S absent } \\
\text { S present }\end{array}$ & $\begin{array}{l}27.59 \\
25.78\end{array}$ & 1.81 & $\begin{array}{l}30.88 \\
23.74\end{array}$ & 7.14 & $\begin{array}{l}27.00 \\
20.73\end{array}$ & 6.27 & $\begin{array}{l}28.48 \\
23.21\end{array}$ & 5.27 \\
\hline
\end{tabular}

reinforcement stimulus present, and 4 with the startle stimulus presented in the absence of the secondary reinforcer. The test trials were arranged in $A B B A B A A B$ sequence, "A" signifying a secondary reinforcement trial for approximately half the rats and a non-secondary reinforcement trial for the other. The preocedure, including the temporal sequences, was identical for Group C. Of course, the light-sound stimulus for this group presumably did not function as a secondary reinforcer, as there had been no previous pairing with delivery of food. The ITI during testing was $50 \mathrm{sec}$., the mean of the 4 intertrial intervals used during training. The sound of the paper drive mechanism was present throughout all phases of the experiment.

\section{RESULTS}

It was predicted that the suppression of startle in the jresence of the light-sound stimulus would be greater for Group E, for which this stimulus was presumably a secondary reinforcer, than for Group C, for which it was not. The results, presented in Table 1 , show that this was the case.

For each test day and for all 3 test days combined, each $\mathrm{S}$ was assigned a score which was the difference between the mean startle response in the absence of the light-sound stimulus and the mean startle response in the presence of this stimulus. U-test analyses of these difference scores revealed a significant effect on Test Day $1, \mathrm{U}(18,20)=101, \mathrm{p}<.02$ and over Test Days 1-3, $U(18,20)=111, p<.025$, supporting the hypothesis.

\section{DISCUSSION}

The data here reported support the view that the decrement in startle observed in the presence of an extraneous visual-auditory stimulus is greater if that stimulus had been previously associated with the receipt of food (was presumably a secondary reinforcer) than if it had not been so associated with food. Unlike the study of Armus et al (1964), the effect was greater on the first day of testing than on later testing days.

The studies of Brown, Kalish, \& Farber (1951) and Meryman (1952) show that rats startle more when fearful than when less fearful and when hungry than when less hungry, i.e., when under relatively high as opposed to relatively low drive. Reasoning from these studies, the present results can be interpreted as indicating that the presence of a secondary reinforcement stimulus lowers the general drive level. If so, this mechanism may be basic to the reinforcing properties of conditioned reinforcers in general.

Granted that the depression of startle in the presence of the light-sound stimulus was greater when this stimulus had been previously associated with the receipt of food, one can still maintain that this greater decrement resulted from postural adjustments or other anticipatory responses that the animals assumed when the stimulus was presented, rather than from secondary reinforcing properties of the stimulus. However, the construct of secondary reinforcement is not necessarily limited to central nervous system activity. It may well be that the secondary reinforcing properties of a stimulus are dependent, at least in part, on feedback from such postural adjustments, fractional anticipatory responses, and other peripheral reactions to the stimulus. A similar analysis can be applied to the view that the obtained difference, although resulting from the secondary reinforcement properties of the stimulus, involved postural adjustments, etc., rather than a reduction in general drive. Peripheral responses may be heavily involved in what is termed "drive." The generality of the findings would, of course, be extended if responses other than whole body startle were to show similar effects.

\section{References}

Amus, H. L., Carlson, K. R., Guinan, J. F., \& Crowell, R. A. Effect of a secondary reinforcement stimulus on the auditory startle response. Psychol. Rep., 1964, 14, 535-540.

Brown, J. S., Kalish, H. I., \& Farber, I. E. Conditioned fear as revealed by magnitude of startle response to an auditory stimulus. J. exp. Psychol., 1951, 41, 317-328.

Hoffman, H. S., \& Fleshler, M. Startle reaction: Modification by background acoustic stimulation. Science, 1963, 141, 928-930.

Meryman, J. J. The magnitude of startle response as a function of hunger and fear. Unpublished master's thesis, State University of Iowa, 1952.

Trapold, M. A. The effect of incentive motivation on an unrelated reflex response. J. comp. physiol. Psychol., 1962, 55, 1034-1039.

Note

1. This research was partly supported by Institutional Grant No. GU-404 from the National Science Foundation awarded to The University of Toledo Research Foundation. 